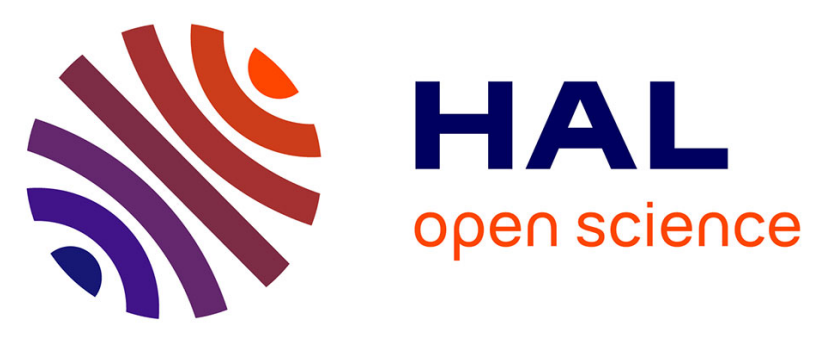

\title{
Integrating Model-Driven Engineering as the Next Challenge for Artificial Intelligence - Application to Risk and Crisis Management
}

Frederick Benaben, Matthieu Lauras, Audrey Fertier, Nicolas Salatge

\section{- To cite this version:}

Frederick Benaben, Matthieu Lauras, Audrey Fertier, Nicolas Salatge. Integrating Model-Driven Engineering as the Next Challenge for Artificial Intelligence - Application to Risk and Crisis Management. WSC 2019 - Winter Simulation Conference, Dec 2019, National Harbor, United States. pp.1549-1563, 10.1109/WSC40007.2019.9004828 . hal-02493057

\section{HAL Id: hal-02493057 https://imt-mines-albi.hal.science/hal-02493057}

Submitted on 18 Jan 2021

HAL is a multi-disciplinary open access archive for the deposit and dissemination of scientific research documents, whether they are published or not. The documents may come from teaching and research institutions in France or abroad, or from public or private research centers.
L'archive ouverte pluridisciplinaire HAL, est destinée au dépôt et à la diffusion de documents scientifiques de niveau recherche, publiés ou non, émanant des établissements d'enseignement et de recherche français ou étrangers, des laboratoires publics ou privés. 


\title{
INTEGRATING MODEL-DRIVEN ENGINEERING AS THE NEXT CHALLENGE FOR ARTIFICIAL INTELLIGENCE - APPLICATION TO RISK AND CRISIS MANAGEMENT
}

\author{
Frederick Benaben \\ Matthieu Lauras \\ Audrey Fertier \\ Nicolas Salatgé \\ Centre Génie Industriel \\ IMT Mines Albi \\ Campus Jarlard - Route de Teillet \\ Albi, 81000, FRANCE
}

\begin{abstract}
Artificial Intelligence (AI) is currently on top of the hype regarding simultaneously research publications and industrial development. However, the current status of AI makes it quite far and different from the current understanding of Human intelligence. One suggestion that is made in this article is that ModelDriven approaches could be considered as an interesting avenue to complement classical visions of AI and to provide some missing features. Specifically, the use of Model-Driven Engineering tools (such as metamodel and model transformation) could benefit to the domain of AI by introducing a way to extend the apprehension of unknown situations. To support that proposal, an illustrative example is provided regarding the domain of risk and crisis management.
\end{abstract}

\section{INTRODUCTION}

Artificial Intelligence (AI) is at the center of most of the main developments in both research in computer science and industrial innovation in software systems. This is an amazing trend of nowadays and out of any cynical "buzz word" consideration, it is obvious that both the research in computer science and the software system industry are being transformed by this emergence. However, one can argue that it has been going very fast (especially due to availability of dormant past results in the domain of neural networks and machine learning) and that what we call AI should actually be consider as quite far from the simple notion of intelligence.

In Legg and Hutter (2007a, 2007b), a lot of definitions of intelligence are studied, including some extracted from AI domain. As a conclusion, the authors tries to provide a synthesis which includes the following statements regarding stable features of definitions:

- "Intelligence is seen as a property of an individual interacting with an external environment, problem or situation" which includes an idea of perception.

- "intelligence is related to the ability to succeed" which implies the existence of objectives.

- "there is a strong emphasis on learning, adaptation and experience" which requires knowledge.

In addition, in this article, the following definitions will be considered for intelligence as well:

(1) The ability to learn or understand or to deal with new or trying situations (merriam-webster.com)

(2) the ability to apply knowledge to manipulate one's environment or to think abstractly as measured by objective criteria (merriam-webster.com) 
(3) the ability to perceive or infer information, and to retain it as knowledge to be applied towards adaptive behaviors within an environment (wikipedia.org)

(4) the ability to acquire and apply knowledge and skills (oxforddictionaries.com)

(5) the ability to learn, understand, and make judgments or have opinions that are based on reason (dictionary.cambridge.org)

(6) the ability to use memory, knowledge, experience, understanding, reasoning, imagination and judgment in order to solve problems and adapt to new situations (AllWords Dictionary).

From these definitions, four main characteristics can be considered as the root of intelligence:

- Perception: directly connected to the first statement from Legg and Hutter, and corroborated by the use of words such as "learn", "understand", "perceive or infer information", this basically means that intelligence requires to be able to gather data about the context of the faced situation.

- Interpretation: this idea is not provided by the analysis of Legg and Hutter but it is a consequence of the previous idea of "perception" (and probably included in their first statement). In this article, the gathering of signals from the situation and the understanding of these signals are to different points: perception for the first one and interpretation for the second one.

- Exploitation: similar to the second statement of Legg and Hutter, this idea implies that there are objectives to reach by using the perceived and interpreted signals. The use of words like "apply", "make judgment or have opinions", "solve problems" confirm this statement. This would often be a decision to take, with the support of the situation understanding.

- Knowledge: echoing the third statement of Legg and Hunter, another principal idea regards the capitalization of experience. This is also embedded in the notion of decision tacking. The previous definitions show that trend with the use of terms like "apply knowledge", "retain it as knowledge", "based on reason", "memory, knowledge, experience".

Based on these considerations, this article considers the following definition of "intelligence":

Definition 1 Intelligence can be seen as the ability to use one's perception senses to construct an understanding of any given (and potentially unknown) situation and to confront it to an existing stored knowledge to enact optimally, according to objectives.

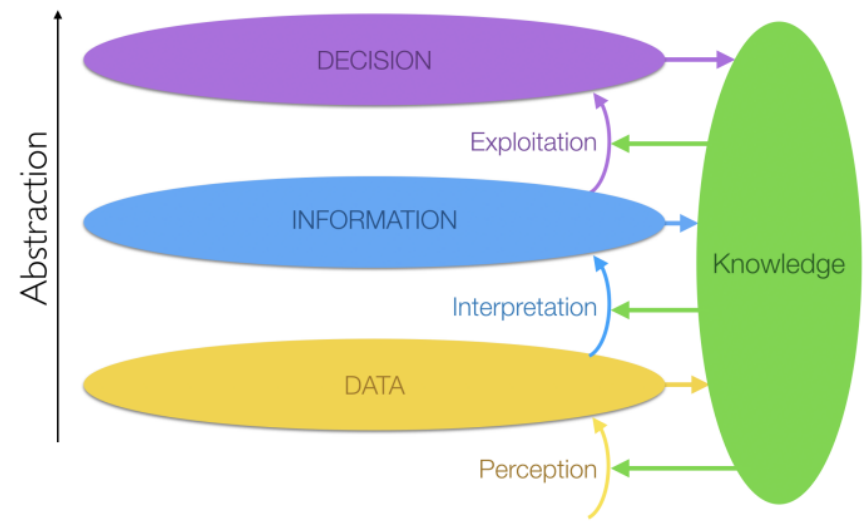

Figure 1: Big Picture of the definition of Intelligence suggested and used in this article.

Figure 1 shows the idea of climbing abstraction levels from gathering signals about the situation (data level) to understanding that situation with regards to an existing knowledge (information level), in order to take decisions based on the understanding of the situation and the stored knowledge. Basically, the 
knowledge is used at every level and could be enriched by all levels. This vision of intelligence is quite close to the results presented in (Rowley 2007) as the wisdom hierarchy or the DIKW hierarchy (where D stands for Data, I for Information, $\mathrm{K}$ for Knowledge and W for Wisdom).

The following of that article discusses in section 2 how model-driven engineering can be used to complete traditional AI tools to provide a more relevant intelligence, while section 3 illustrates this framework within a use-case of risk and crisis management.

\section{TOWARDS A MORE INTELLIGENT AI USING MODEL-DRIVEN ENGINEERING}

\subsection{Classical Artificial Intelligence approaches}

Artificial Intelligence is mainly based on Data Science. According to (Jagadish et al. 2014), Data Sciences are mainly based on Data Management and Data Analytics. On the one hand Data Management includes Acquisition, Content Extraction and Data Integration and Representation. On the other hand, Data Analytics concerns Analysis and Human-Based Interpretation. This human-based interpretation is not to be confused with the interpretation mentioned as the transition to the second level (Information) of the intelligence framework presented in section 1. With regards to the framework presented in the previous section and illustrated through Figure 1, it is easy to see that the Data Management step is dedicated to perform the perception feature. The Data Analytics is dedicated to provide the user with a content that can be interpreted by him to take decision: the Analysis sub-step focuses on detecting patterns and correlations (for statistical purpose) to feed the Decision level for the Human-Based Interpretation.

Figure 2 illustrates Data Science on the Intelligence framework introduced in Figure 1:

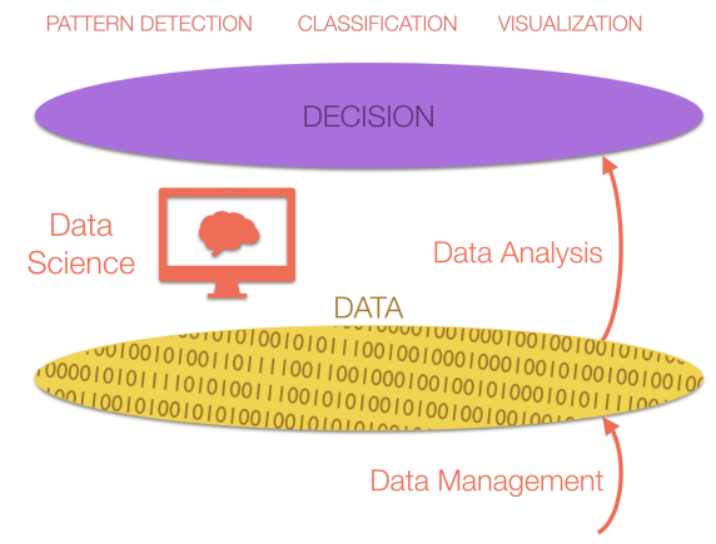

Figure 2: Location of Data Science in the Intelligence Framework.

Basically, the most important finding from that analysis is that Data Science and more broadly general approaches of AI actually do short-cut the Information level. These approaches focus on providing the decision level with material that would actually support the making of a decision by establishing statistical reports on the data. This definitely allows such systems to deal with Volume and Velocity of data.

\subsection{Model-Driven Engineering}

Model-Driven Engineering (MDE), as described in (Da Silva 2015) mainly relays on two basic activities: Modeling and Model transformation. It is important to notice at this point that MDE is absolutely not dedicated to software engineering (though it is used a lot in that domain), and as stated in (Dietz et al. 2014) can be used in Industrial Engineering contexts. Modeling can be defined as: 
Definition 2 Modeling is the activity of building a representation of a known or unknown entity according to shared concepts and vocabulary.

In an operational context, modeling is dedicated to the following objectives (which may obviously be combined, depending on the context):

- Modeling to manage the behavior (simulation): exploiting models to anticipate on the behavior of a subject (e.g. weather models, heat diffusion equations, etc.).

- Modeling to build appropriately (conception): defining the static and dynamic structure of a system to support its construction (e.g. furniture plan, algorithm, etc.).

- Modeling to explain or understand (communication): describing a system to make it understandable (e.g. marketing presentation of a product, meeting schema, etc.).

Besides, one can notice that the three previous usages of models can also be considered with regards to the formal and rigorist degree of the models: simulation requires very formal models, communication usually exploits more informal models, while conception is in between and can use hybrid models.

Figure 3 illustrates schematically but very accurately the activity of modeling (independently from the domain, the subject, the objectives or the type of the concerned modeling):

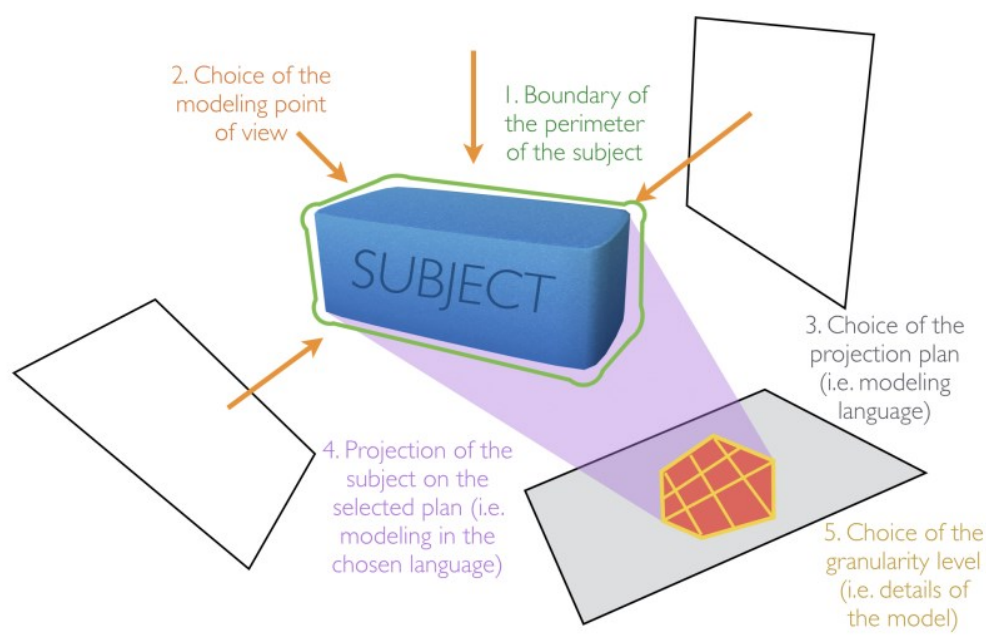

Figure 3: A generic representation of the modeling activity.

This vision of modeling is based on the following steps:

1. Boundary of the subject: definition of the exact perimeter of the system to be modeled.

2. Choice of the modeling point of view: definition of the why, for whom and for what purpose of the modeling activity.

3. Choice of the projection plan: selection of the modeling language that fits the point of view (basically that is relevant to the goal of the model and understandable by the future users).

4. Projection of the subject on the projection plan: this is the model building step.

5. Choice of the granularity level: definition of the appropriate precision level of the model.

One important remark concerns the question of consistency between point of views. Actually, it is very common to have to realize several models of the same system (plan of a building VS energetic behavior VS budget and costs). It is so very important to understand, especially in "conception" that any choice on 
one point of view has some impacts on other points of view: esthetic and aerodynamic considerations regarding the design of a car must take into account the plans of the engine, otherwise there is a major of risk of the engine not fitting under the hood...

It is important to notice that step 4 requires a metamodel. There are lot of definition of what is a metamodel. (OMG 2017) describe it as "a model that defines the language used to define a model", it is so the model of a modeling language. More pragmatically, a metamodel can be seen as "the description of all concepts of a language, their semantics and the syntactic related to the use of these concepts" (Chapron 2006). Based on this and on (Bezivin 2005), we will keep the following definition:

Definition 3 A metamodel is the description of concepts and relationships between these concepts that allows to defines models where: the models conforms to the metamodel and the metamodel is represented by the models (NB: a metamodel is usually the high-level structure of an ontology).

Figure 4 illustrates the classical abstraction layers of modeling/metamodeling:

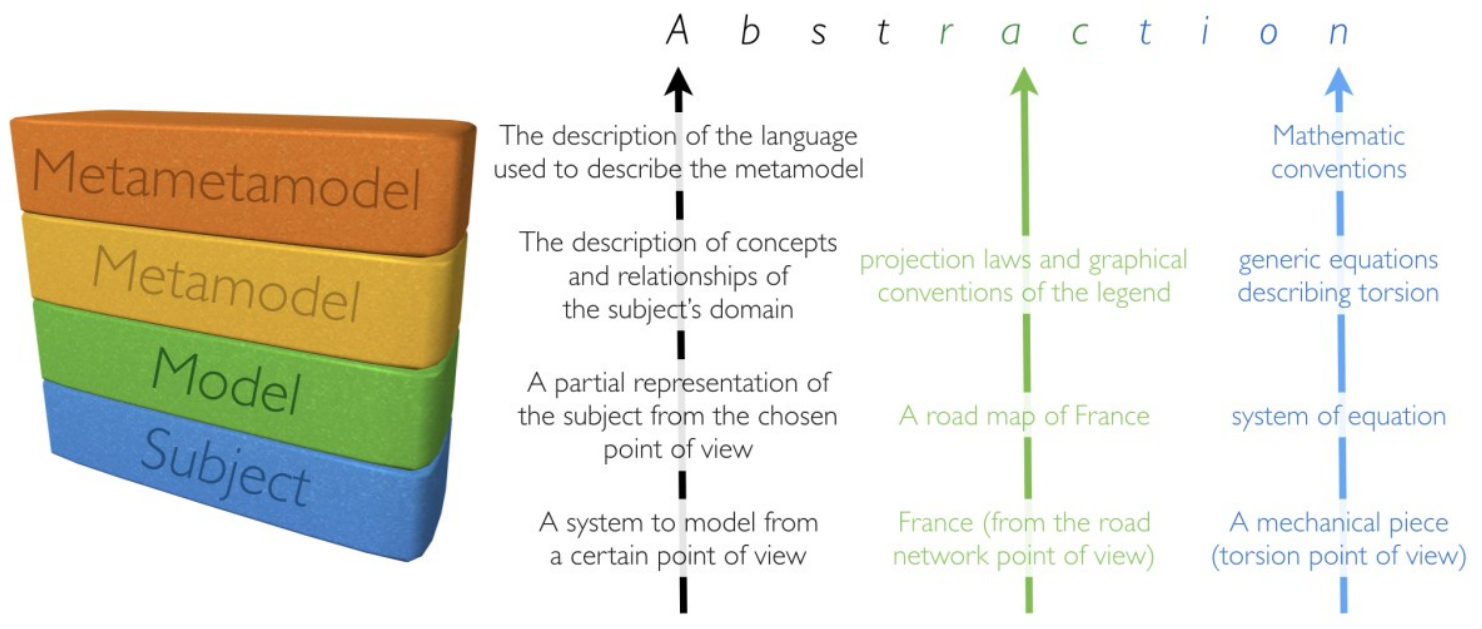

Figure 4: the notion of metamodeling.

The concept of metamodel is particularly pregnant in the domain of model transformation. Transformation rules are actually defined between metamodels. On a very schematical point of view model transformation is the tool used to travel the model-driven engineering cycle. For instance the cascading Model-Driven Architecture provides transitions between Computer Independent Model (CIM, mostly representing requirements and expectations of a software) and Platform Independent Model (PIM, mainly figuring the theoretical system responding to the CIM), and then between PIM and Platform Specific Model (PSM, representing the concrete vision of the implementation of the PIM on a chosen platform). Each of these transitions is supposed to be assumed by model transformations, describing transformations rules between source metamodels and target metamodels.

The objective of a model transformation is to exploit the knowledge embedded in a source model to create (systematically and thus, potentially automatically a target model. The source model is built according to a source metamodel (which defines modeling rules for the source domain). The target model, similarly, must conform to a target metamodel (which defines the target domain). A key principle is that the two metamodels, source and target, should not be totally disjointed in order to share a number of concepts (as shown in Figure 5). Because of this common conceptual space between the source space and the target space, a set of rules can be established. These rules must describe how the concepts of the target metamodel (the ones located in the common space) can be obtained. from the concepts of the source 
metamodel (also located in this common space). These rules will constitute the transformation rules (or mapping rules). Considering the existence of these concepts shared between metamodels, the source model contains modeling elements resulting from these shared concepts (the shared part of the source model) and others resulting from the specific concepts (the specific part of the target model ). From this source model we will then be able to identify a capitalized knowledge (corresponding to the material resulting from the specific part) and an extracted knowledge (resulting from the shared part). The knowledge extracted will concretely constitute the raw material for the transformation of the model strictly speaking whereas the capitalized knowledge will rather be stored for later use. The mapping rules can then be applied to the extracted knowledge to transform the elements of the source model resulting from the shared concepts into elements of the target model also referring to these shared concepts. The result of this operation is the obtaining of a transformed knowledge. Additional knowledge must then be imported in order to fill the gaps concerning the specific concepts of the target metamodel (since only the shared concepts have been covered by the application of the mapping rules). The transformed and additional knowledges are combined to create the shared part and the specific part of the target model. Figure 5 illustrates schematically the mechanism of model transformation:

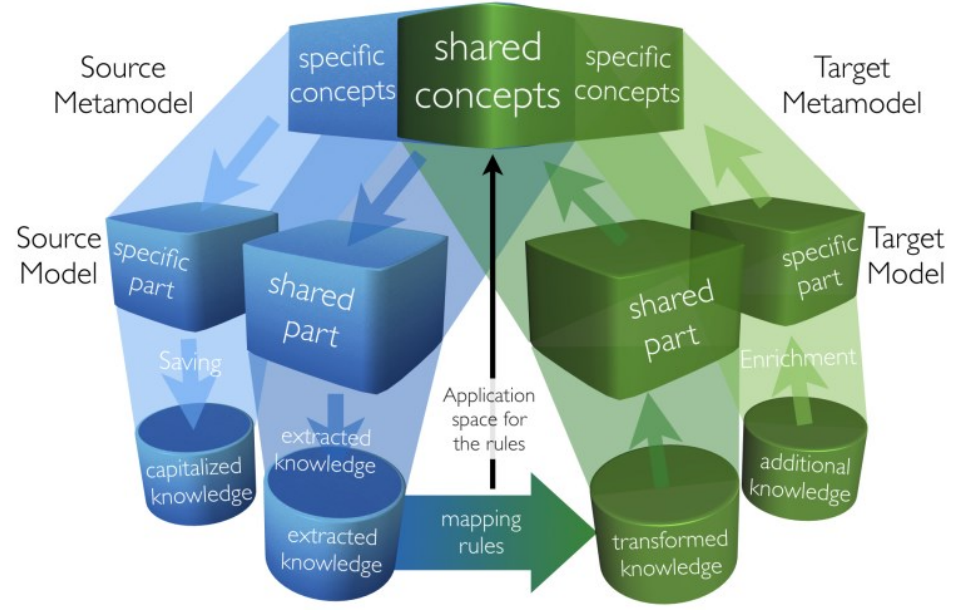

Figure 5: Model transformation basic principle.

It should of course be noted that these different "knowledges" may be more or less consistent depending on the relevance and quality of the transformation of models. If the capitalized knowledge and the additional knowledge are not significant (compared to the knowledge extracted and transformed), one can suppose that the considered transformation of models is relatively convincing (in the sense of the potential of automation). On the other hand, if the extracted knowledge and the transformed knowledge are weak compared to the capitalized and additional knowledge, it is very likely that the domains considered are too distant and that the transformation turns out to be very manual (i.e. not programmable by generic rules). The first of the two "asymptotic" (i.e. at the limit) situations associated with these considerations corresponds to a transformation of models between two conceptually disjoint domains (called pure exogenous), which would in fact be a creation of a target model on the basis of the only additional knowledge (the transformed knowledge being empty because of the disjointed nature of the two spaces of departure and arrival). It would simply be a "from scratch" achievement of the target model. The second "asymptotic" situation corresponds to a transformation between two conceptually confused domains (socalled endogenous), which would concretely result in a creation of the target model on the basis of transformed knowledge alone (the additional knowledge being empty due to the confused nature of the two spaces of departure and arrival). It would ultimately be a transformation of a first model into a second model 
rigorously equivalent, but expressed in a different language (for instance the transformation of a process model expressed as an Petri net into a BPMN model).

Moreover, a transformation is called "vertical" if it involves different levels of abstraction within the model-driven engineering cycle (typically, the transformation of PIM to PSM in an MDA cycle is a vertical transformation). Similarly, a transformation is said to be "horizontal" if it remains at the same level of abstraction within the model-driven engineering cycle (the transformation of a GRAI digraph into a UML activity diagram presented in (Grangel et al. 2010) is a horizontal transformation). Thus, by freely taking inspiration from (Combemale 2008), Figure 6 describes a summary table aiming at linking the orientation of model transformations (horizontal or vertical) with the origin of model transformations (endogenous or exogenous) with regards to their frequency of occurrence:

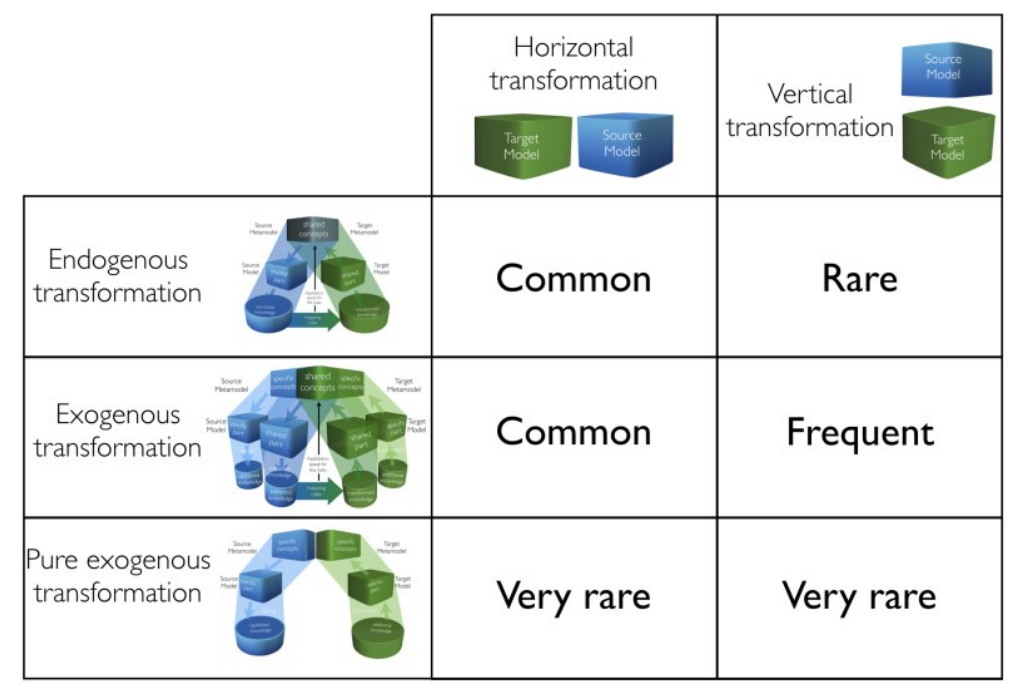

Figure 6: Frequency of the different model transformations.

Finally, regarding MDE, the question about the location of MDE on the intelligence framework presented in Figure 1 should be considered to echo the proposal of Figure 2. With regards to that intelligence framework, it can be stated that the Modeling step (based on metamodel) is dedicated to perform the interpretation feature. The Model Transformation step is dedicated to use the obtained model to generate new content that would be more useful for the user to take decision. This is the exploitation feature

Figure 7 illustrates the location of MDE on the introduced Intelligence framework:

FORMAL ANALYSIS SIMULATION GALCULATION

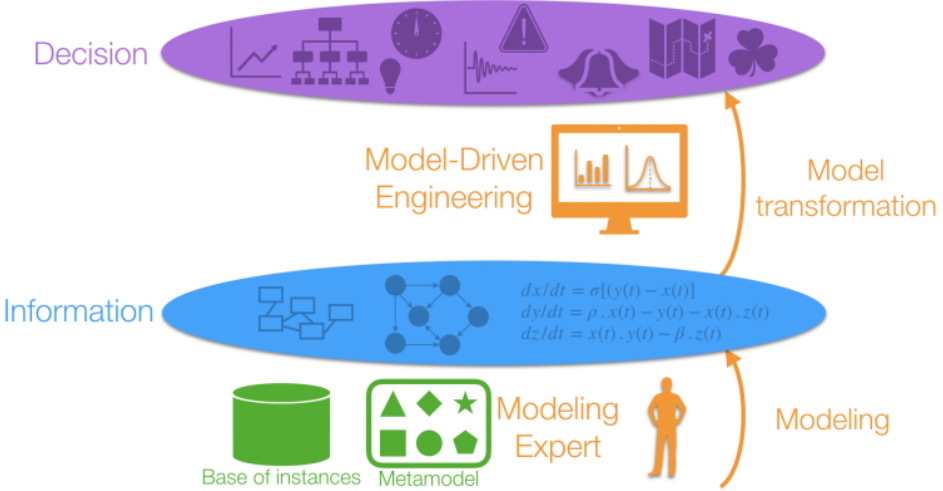

Figure 7: Location of Data Science in the Intelligence Framework. 
Basically, the most important finding from that analysis is that Model-Driven Engineering actually do not consider the data level (and the perception feature) out of human consideration. This is basically the human modeler that must make the link between signals he or she can gather from the observed situation and concepts of the metamodel to be instantiated. Then, the obtained model(s) can be exploited automatically through model transformation for decision support. This definitely allows such systems to deal with Variety and Value of data. It is also important to notice that the obtained model(s) can also be used in various types of other exploitation types (usually dedicated to decision support but not only) like visualization or simulation. Visualization is not necessarily based on generated models and can be obtained directly from data. However, simulation absolutely requires formal models to be performed.

\subsection{Complementarity and limits of both approaches}

Based on both conclusions of subsections 2.1 and 2.2, and if we consider that the question of Veracity of data is out of scope due to the fact that we only consider data that is already clean and trustable (the topic is not about data source discovery or data filtering and cleaning), then, it is easy (and potentially too simple) to state that data science could take care of Volume and Velocity, will model-driven engineering could tackle Variety and Value. In any case, both approaches struggle with specific conditions as soon as we extend the range and the amount of data to manage:

- Data science can not deal with unknown type or content: just like a shark, able to detect a drop of blood in millions of litters of sea water, but which would be lost with a Rubik's Cube $\AA$. This is due to the fact that the data associated to the Rubik's Cube ${ }^{\circledR}$ is out of the scope of its perception and it can only apply a default behavior (like attack, ignore or escape).

- Model-Driven Engineering can not manage a large amount of data: just like a baby, able to start to play with a Rubik's Cube $®$ because of its shape, color, touch, etc. but would be unable to detect a drop of lemonade in his bath among all other tastes and odors of the water.

These considerations have been largely discussed in (Benaben et al. 2016) and are represented on Figure 8 in a general perspective mapped on the framework of Figure 1:

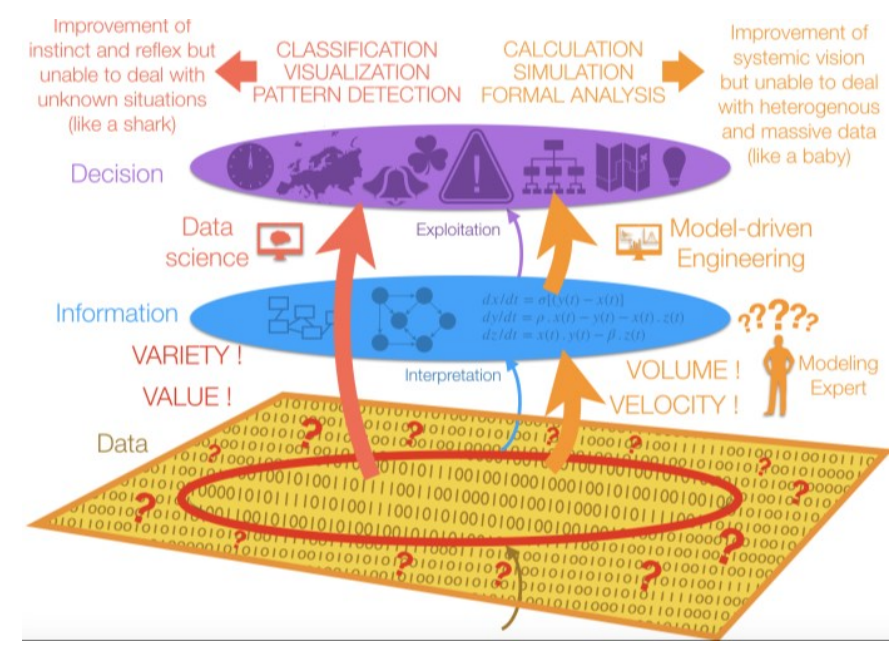

Figure 8: Limits of both Data Science and Model-Driven Engineering in the Intelligence Framework.

The line of attack is, first, to use Data Science to perform the modeling step by interpreting the flow of incoming data thanks to Data Analysis tools. Then it is expected to instantiate concepts of a metamodel to create situation model(s). Second, the goal is to use Model-Driven Engineering to exploit the generated 
situation model(s) by taking advantage of the content of the models through Model Transformation tools. Then it is expected to obtain more relevant and more actionable models to support decision making.

Figire 9 illustrates how, considering the previous element, Data Science and Model-Driven Engineering can be located altogether on the Intelligence framework of Figure 1. The upper left part of Figure 9 concerns Data Science (Data Analytics and Data Management), the upper right part concerns Model-Driven Engineering (Modeling and Model Transformation) and the bottom part represents the way both could be combine to provide an Artificial Intelligence framework:

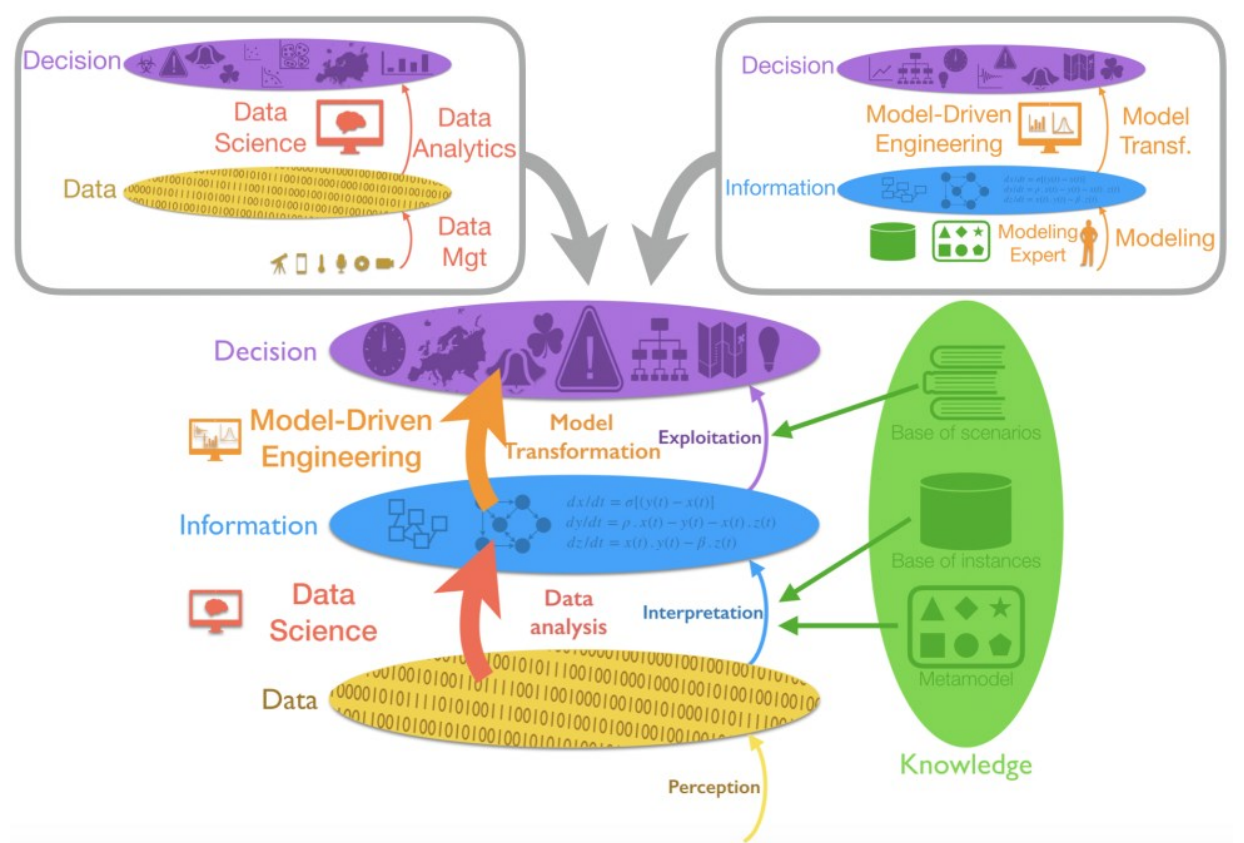

Figure 9: The Artificial Intelligence proposal Framework.

\section{IMPLEMENTATION OF THE ARTIFICIAL INTELLIGENCE FRAMEWORK ON A RISK AND CRISIS MANAGEMENT USE-CASE}

\subsection{Rapid description of the Loire Flooding Use-Case}

The Loire flooding is one of the three most feared disasters on the French Territory (with the Seine flooding and an earthquake on the French Riviera). The Loire river is characterized by significant variations in flows (DREAL 2016) and its significant rates of containment and urbanization. In 1856, such a flood had flooded 110,000 hectares where there are today more than 300,000 people, 13,600 companies and 72,000 jobs (DREAL 2016). To enable the simulation of such a scenario, we have collaborated with the Loire-CherIndre and Maine-Loire Services of Forecast of Floods (In French SPC). Two models of propagations have been developed: a five-year flood and a 100-year flood on the Middle Loire, between the Bec d'Allier and the Bec de Maine. In this example, we focus on a 100-year flood event that corresponds to the highest known flood event for a given region (MEDD 2004).

A hydro-meteorological scenario, reported in (Dolidon 2016), realistically models the water inflow of the six tributaries present in this part of Loire in the case of a 100-year flood. Thus, three daily flow and water level (coast) surveys were developed for the seven reference hydrometric stations and the 11 expected flood days. Each survey contains forecast flow and coast over the next 56 hours and three probability levels: high, median and low. 


\subsection{Metamodel for crisis management}

As described in (Lauras et al. 2015), the COSIMMA (Collaborative Situation MetaModel of Albi) defined for crisis management is structured according to two layers: (i) the core layer which actually describes concepts and relations of any collaborative situation, and (ii) the specific layer containing concepts that inherit from the core concepts and that describe more precisely concepts of the domain. The core is agnostic while the layer is dedicated to crisis management domain (in some other research works, other layers have been defined about supply chain and health care.

The core layer of the metamodel has been organized in five complementary parts:

- Context system (light grey) including components and characteristics of the considered environment, and also opportunities or threaten specific to these environment characteristics.

- Objective system (medium grey) containing characteristics of collaborative network (common objective and facts that the collaboration has to manage).

- Partner system (strong grey) expresses the different resources and know-how of the partners. This includes notably capabilities, patterns, instructions, resources (information, material, people, etc.), flows (linking capabilities) and mediator able to orchestrate the different business processes.

- Behavior system (dark grey) describes the concrete operations, which are deployed to concretize the collaboration. This includes business processes / activities.

- Performance system (white) assesses the overall performance of the collaboration by comparing dedicated Key Performance Indicators (KPIs) to the measures on the field.

The crisis management metamodel is structured as presented in Figure 10.

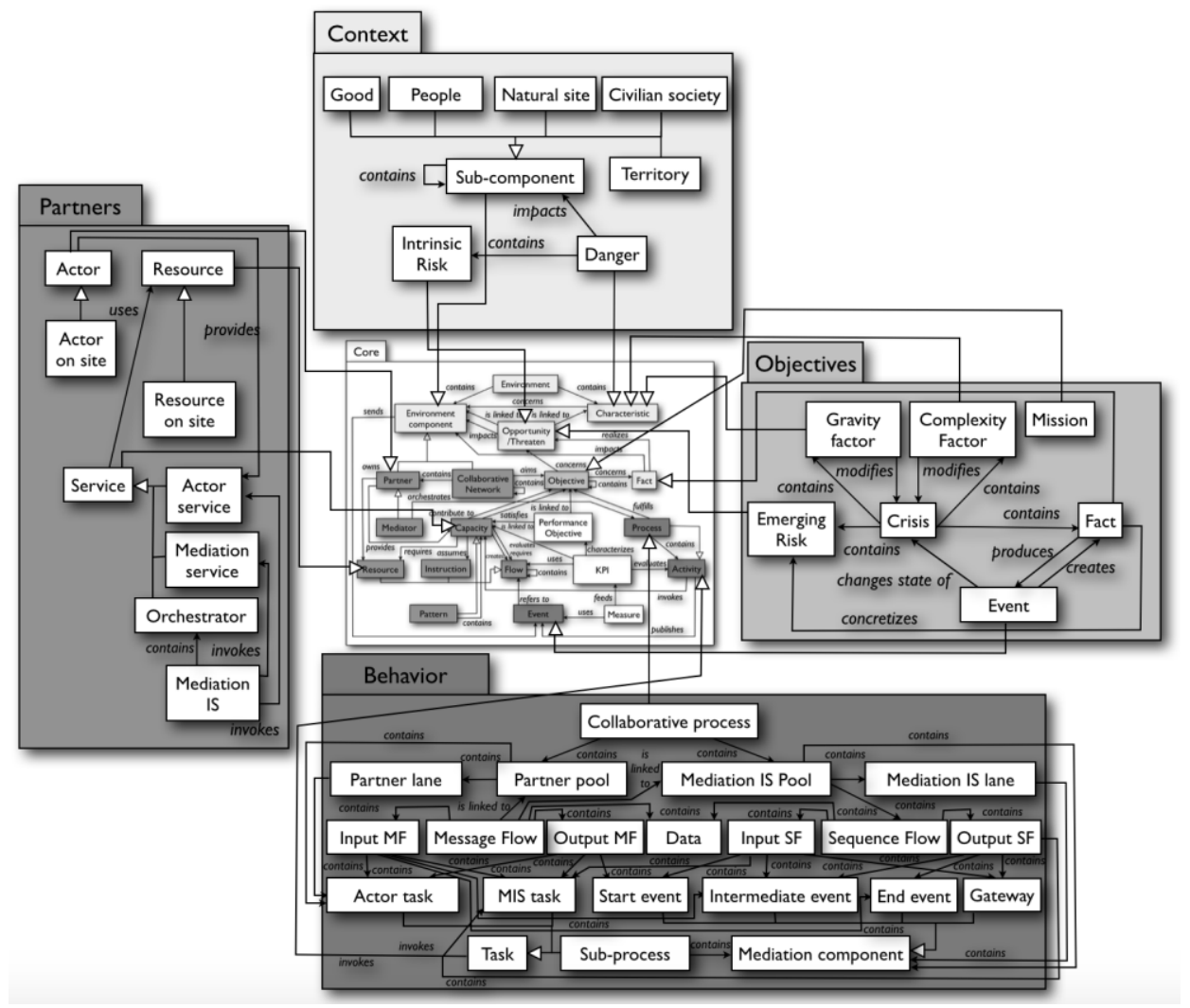

Figure 10: The Collaborative Situation MetaModel of Albi (COSIMMA). 
The core metamodel at the center and four packages (Context, Partners, Objectives and Behavior) contain concepts dedicated to crisis situation inheriting from the core concepts. In the following, only concepts dedicated to interpretation of data (Context, Partners, objectives packages) are described as they are currently implemented in the knowledge base:

Context package:

- Good: any human-made elements (e.g. building, road, etc.).

- People: any group of person (e.g. students of a school, employees of a plant, etc.).

- Natural site: any natural element of the environment (e.g. lake, forest, etc.).

- Civilian society: any social actors (e.g. media, intellectual society, etc.).

- Territory: any administrative area (e.g. county, island, etc.).

- Danger: any specific dangerous characteristic of the environment.

- Intrinsic risk: any permanent risk due to identified danger (e.g. earthquake, riot, etc.).

Partners package:

- Actor: any stakeholder involved in crisis management (e.g. firemen, EMS, etc.).

- Resource: any resource used by actors (e.g. truck, decontamination tent).

- Service: any capability of actors (e.g. evacuate victims, treat injured people, etc.).

- Actor service: any service specifically provided by actors.

- Mediation service: any service provided by Mediation IS.

Objectives package:

- Emerging risk: any risk specifically emerging due to the crisis itself.

- Effect: any direct consequence of the crisis itself (e.g. 10 injured people, fire, etc.).

- Mission: any objective directly responding to identified risk or effect of the crisis.

- Event: any event occurring during crisis management that must be considered as triggering.

- Gravity factor: any characteristic of the current situation that may change the gravity of the crisis.

- Complexity factor: any characteristic of the situation that may change the type of the crisis.

\subsection{Illustration with the RIOSUITE software tools}

The following describes the way the RIOSUITE suite of tools is used to deal with crisis management, implementing the Artificial Intelligence framework presented above and using the metamodel presented in the previous section 3.2. The presented screenshots do show the way RIOSUITE climbs the abstraction levels of the Artificial Intelligence Framework: (i) Perception, i.e. data gathering, (ii) Interpretation, i.e. from data to information, and (iii) Exploitation, i.e. from information to decision.

\subsubsection{Perception - data gathering}

The perception aims at collecting data related to the faced crisis situation. In the case of the Loire flooding, considering that there is actually no real crisis, the Loire flooding is simulated through the use of simulation sensors, sending "fake" data extracted from the Services of Forecast of Floods and replicating exactly the data from the scenario they defined. In Figure 11, we can see a screenshot describing on the left part the map of the area with the location of "fake" sensors and on the right part the curves of water level and water flow that the sensors are actually sending. The data sent is respecting the WSN (web-service notification) standard. 


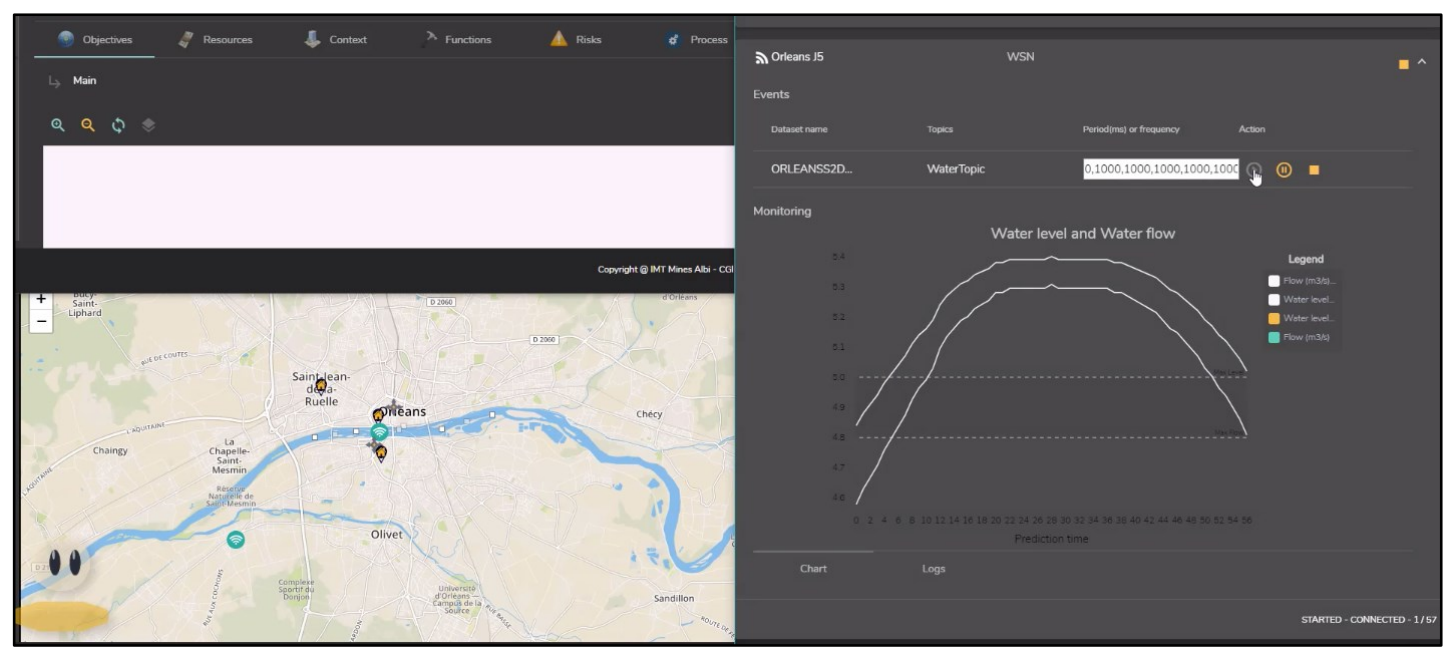

Figure 11: The Perception level in RIOSUITE.

\subsubsection{Interpretation - from data to information}

The interpretation is dedicated to automatically build the situation model at the information level, i.e. to instantiate concepts of the metamodel related to context, partners and objectives.

For the context, the mechanism is simply based on extracting information from OpenStreetMap ${ }^{\circledR}$ based on a pre-filtered selection of amenities. For the partners, this is mainly by interviews of the stakeholders that the actual capabilities (and their relationships) can be identified. Regarding the objectives, the used mechanisms are based on formal rules that have been described in (Fertier 2018). These rules are dedicated to detect patterns and to instantiate dangers mainly. Then the RIOSUITE system identify (thanks to the context model) the stakes that are in the area of the danger and generates risks according to the susceptibility of these stakes to that danger. Figure 12 illustrates the objective interpretation. On the upper left part of the picture are the risks that have been interpreted from the presence of buildings in the area of the danger of flooding identified thanks to the triggering of some rules due to the values of some sensors. On the bottom left part are these risks on the map. On the right are the curves representing the sent data through the "fake" sensors.

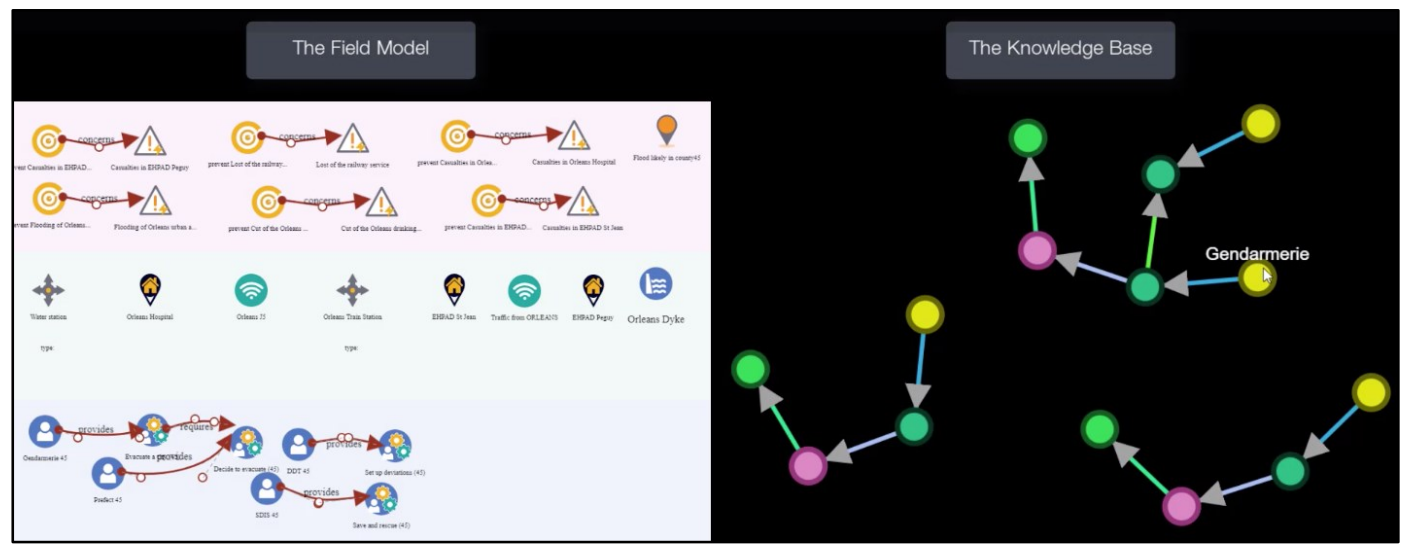

Figure 12: The Interpretation level in RIOSUITE. 
On a practical point of view, the complete obtain models is similar to the next Figure 13 where on the left part are the three models (from up to bottom: objectives, context, partners) and on the right part is the graph database representing the embedded knowledge base.

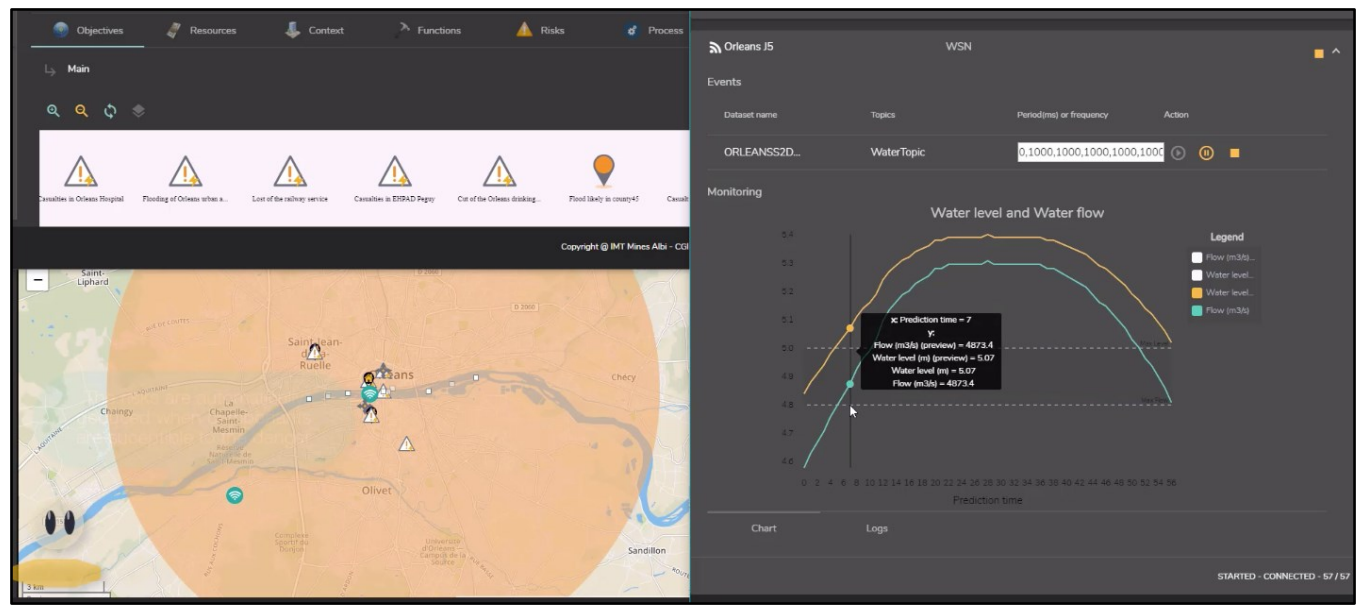

Figure 13: The obtained models from the interpretation level in RIOSUITE.

\subsubsection{Exploitation - from information to decision}

The exploitation is dedicated to use the obtained models (at the information level) to provide decision support to the crisis managers. Basically, as presented on Figure 14, there are three main features at this level. First, the RIOSUITE platform is used to deduced a collaborative process model (see bottom-left part of Figure 14). This is based on a model transformation from the models of context, partners and objectives to a model of behavior. This model transformation has been completely described in (Mu et al. 2016). Second, there is an orchestration engine able to run the obtained collaborative business process among the partners as personalized to do lists (see the upper-left part of Figure 14). Third, there is an agility management tool in charge of the detection of any disruption with regards to the expected situation by continuously comparing the expected situation and the field situation (see the right part of Figure 14).

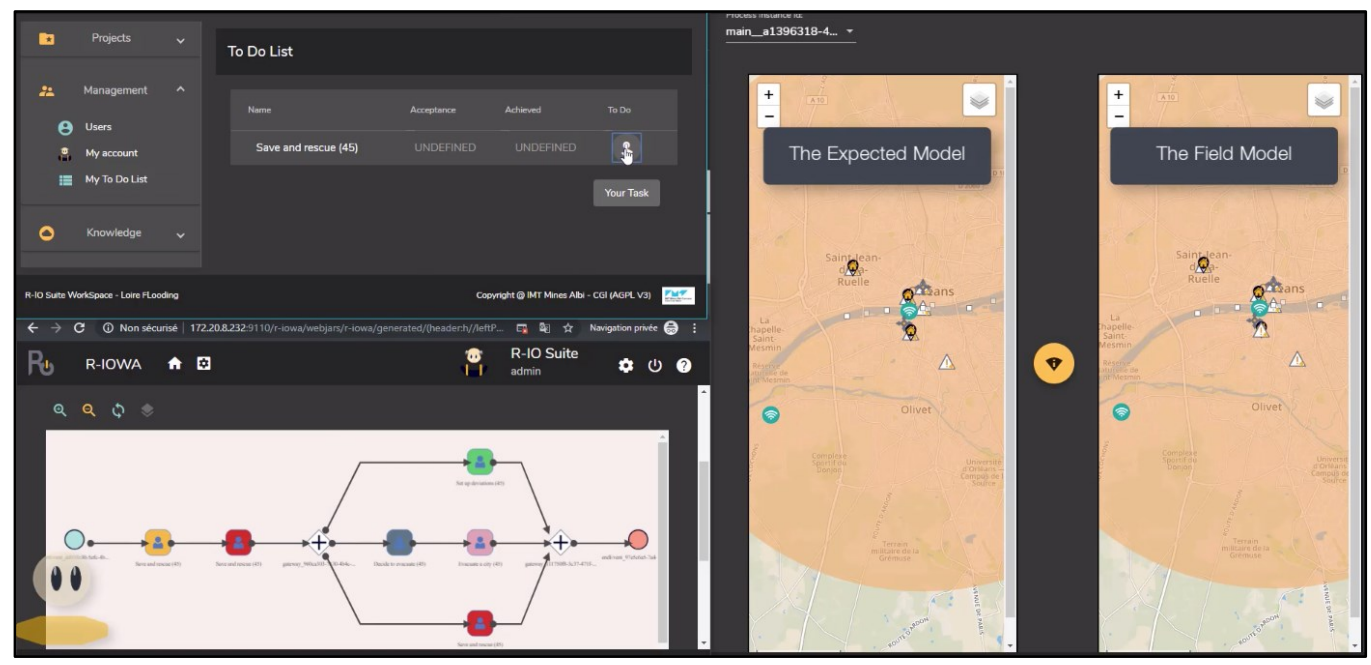

Figure 14: The exploitation level in RIOSUITE. 
The main benefits illustrated through the previous example basically concern the inclusion of models between data gathering and decision support. Thus the proposed approach is able to deal with potentially unknown situations (by casting raw data into concepts) and to use a formal material (the models) to perform complex reasoning and decision support.

\section{CONCLUSION}

This article tried to show how the evolution of Artificial Intelligence could benefit on the integration of Model-Driven Engineering principles. The mechanisms provided by the use of metamodel and models during a decision making process based on artificial intelligence could definitely bridge the gap between the current vision of AI on the one hand, mainly based on data science, and that the conclusions of this article trend to see like "Artificial Reflex" or "Artificial Instinct" more than an actual Artificial Intelligence, and, on the other hand, the expectations of an AI able to deal with more complex situation. The illustration presented in the third section, based on a risk and crisis management scenario have shown how much this hybrid vision, mixing data science and model-driven engineering could be worth.

Finally, on a broader perspective, this article can lead to the following bet: just like big-data brought artificial intelligence back on the foreground, the next achievement of artificial intelligence, particularly in the domain of complex decision making, could bring model-driven engineering back on the stage.

\section{REFERENCES}

Benaben, F., A. Montarnal, A. Fertier, and S. Truptil. 2016. "Big-Data and the Question of Horizontal and Vertical Intelligence: A Discussion on Disaster Management". In Proceedings of the 2016 Working Conference on Virtual Enterprise, edited by H. Afsarmanaseh, L. Camarinha-Matos and A. Lucas Soares, 156-162. IFIPAICT, 480, Springer.

Bezivin, J. 2005 “On the Unification of Power of Models". Software \& System Modeling 4(2):171-188.

Chapron, J. 2009. L'urbanisme Organisationnel: Méthodes et Aides à la Décision pour piloter L'évolution du Système D'information de L'entreprise. $\mathrm{PhD}$ thesis, Department «Organisation et Modélisatin des Systèmes Industriels », Ecole Nationale Supérieure des Mines de Saint-Etienne, Saint-Etienne, France. URL: https://hal.archives-ouvertes.fr/tel-00796061/ accessed $26^{\text {th }}$ September 2019)

Combemale, B. 2008. L'Ingeniérie Dirigée par les Modèles (IDM) - état de l'art”. Internal Report IRIT (UMR CNRS 5505). URL: http://hal.archives-ouvertes.fr/hal-00371565/, accessed $16^{\text {th }}$ September 2019.

Da Silva, A. R. 2015. "Model Driven Engineering: A Survey Supported by the Unified Conceptual Model.” Computer languages, Systems and Structures 43:139-155.

Dietz, J., E. Proper, and J. Tribolet. 2014. The Enterprise Engineering Series. Ed. M. Lankhorst. Springer-Verlag, Berlin Heidelberg.

Dolidon, H. 2016. Synoptique Scenario GéNéPi. Deliverable of the French funded Project GéNéPi, CEREMA Ouest, Nantes, France. URL: https://research-gi.mines-albi.fr/display/GEN/Ressources+Publiques.accessed $16^{\text {th }}$ September 2019.

DREAL 2016. Un Siècle Sans Crues? $3^{\text {rd }}$ ed. Technical report, DREAL Centre Val de Loire, Orléans, France.

Fertier, A. 2018. Interprétation Automatique de Données Hétérogènes Pour la Modélisation de Situations Collaboratives: Application à la Gestion de Crise. PhD thesis, Department "Centre Génie Industrielé", Albi, France. Ecole Nationale Supérieure des Mines d'Albi-Carmaux.. URL: https://tel.archives-ouvertes.fr/tel-02173328/document accessed 26 ${ }^{\text {th }}$ September 2019.

Grangel, R., M. Bigand, and J.-P. Bourey. 2010. "Transformation of Decisional Models into UML: Application to GRAI Grids”. International Journal of Computer Integrated Manufacturing 23(7):655-672.

Jagadish, H., J. Gehrke, A. Labrinidis, Y. Papakonstantinou, J. Patel, R. Ramakrishnan, and C. Shahabi. 2014. "Big Data and its Technical Challenges". Communication of the ACM 57(7):86-94.

Lauras, M., S. Truptil, and F. Benaben. 2015. "Towards a Better Management of Complex Emergencies through Crisis Management Meta-modelling”. Disasters 39(4):687-714.

Legg, S. and M. Hutter. 2007a. "A Collection of Definitions of Intelligence". Frontiers in Artificial Intelligence and Applications 157:17-24. The Netherlands: IOS Press Amsterdam.

Legg, S. and M. Hutter. 2007b. "Universal Intelligence: A Definition of Machine Intelligence". Minds and Machines 17(4):391444.

Mu, W. , F. Benaben, and H. Pingaud. 2016. "Collaborative Process Cartography Deduction based on Collaborative Ontology and Model Transformation”. Information Sciences 334-335:83-102.

MEDD. 2004. Les inondations. Technical report CEREMA Ouest, Nantes, France. 
OMG. 2017. Meta-Modeling and the OMG Meta Object Facility (MOF). White paper by the OCUP 2 Examination Team. URL: https://www.omg.org/ocup-2/documents/Meta-ModelingAndtheMOF.pdf accessed $26^{\text {th }}$ September 2019.

Rowley, J. 2007. "The Wisdom Hierarchy: Representations of the DIKW Hierarchy". Journal of Information and Communication Science 33(2):163-180. 